\title{
1D-confinement of polyiodides inside single-wall carbon nanotubes
}

\author{
M.Chorro $^{1}$, G. Kane ${ }^{2,3}$, L. Alvarez ${ }^{2,3}$, J. Cambedouzou ${ }^{4}$, E. Paineau ${ }^{4}$, A. Rossberg ${ }^{5}$,
} M. Kociak ${ }^{4}$, R. Aznar ${ }^{2,3}$, S. Pascarelli ${ }^{1}$, P. Launois ${ }^{4}$ and J. L. Bantignies ${ }^{2,3}$

1 European Synchrotron Radiation Facility, Grenoble, France

2 Laboratoire Charles Coulomb UMR 5221, Université Montpellier 2, F-34095, Montpellier, France

3 Laboratoire Charles Coulomb, UMR 5221,CNRS- F-34095 Montpellier, France

4 Laboratoire de Physique des Solides, UMR CNRS 8502, Université Paris Sud, bât. 510, 91405, Orsay, France

5 Helmholtz-Zentrum Dresden-Rossendorf, Institute of Radiochemstry, Dresden, Germany

\begin{abstract}
1D-confinement of polyiodides inside single-wall carbon nanotubes is investigated. Structural arrangement of iodine species as a function of the tube diameters is studied. Evidence for long range one dimensional ordering of the iodine species is shown by $\mathrm{x}$-ray and electron diffraction experiments independently of the tube diameter. The structure of the confined polyiodides is investigated by x-ray absorption spectroscopy. The local arrangement study points out the presence of diiodine $\left(\mathrm{I}_{2}\right)$, triodide $\left(\mathrm{I}_{3}^{-}\right)$and pentaiodide $\left(\mathrm{I}_{5}^{-}\right)$with linear structures. This last point reflects the 1D-confinement of the polyiodides inside the nanotubes.
\end{abstract}

\section{Introduction}

Since their discovery [1], single-wall carbon nanotubes (SWNT) have attracted great interest due to their fascinating physical properties. Indeed, considered as intrinsic conductive one dimensional systems, carbon nanotubes have been involved in development of new electronic devices $[2,3,4]$. Tuning the electrical conductivity is a main issue to achieve such developments. One possible way is the doping of carbon nanotubes via the encapsulation route which consists in the insertion of atoms or molecules in the hollow core of the tubes. As carbon nanotubes possess amphoteric behavior, i.e. they can be electron donors or acceptors, the doping can be achieved with halogen ( $\mathrm{Br}, \mathrm{I})$ or alkali atoms $(\mathrm{Li}, \mathrm{Rb})$ [5-9].

Doping carbon nanotubes with iodine atoms leads to air stable compounds for which the conductivity is significantly improved. Charge transfer occurs from the SWNT to the iodine atoms leading to a p-doping of the tubes and formation of stable polyiodides molecules $\mathrm{I}^{\mathrm{n}-}$ $[9,10]$.

The optimization of the charge doping of the nanotubes goes through the control of the filling rate of the tube (mass of iodine inside a tube), assessment of the nature of polyiodides synthesized (number of iodine atoms per charge) and their organization inside the nanotubes. All these parameters depend on the nanotube diameter. Hence the nature and the organization of polyiodides inside carbon nanotubes have been explored by x-ray diffraction (XRD), Raman spectroscopy, transmission electron microscopy (TEM) and x-ray absorption 
spectroscopy (XAS) [11,12,13]. Recent TEM investigations suggest that the arrangement of the encapsulated polyiodides varies depending on the nanotube diameter from a single chain in narrow nanotubes of about $1 \mathrm{~nm}$, to two and three chains in tubes of $1.20 \mathrm{~nm}$ to $1.40 \mathrm{~nm}$, while a crystalline phase of molecular iodine is obtained in nanotubes larger than $1.45 \mathrm{~nm}$ [14]. However less is known about the intrinsic molecular structure of the iodine chains.

In the present work, our objective is to fully characterize the chain structures confined inside SWNT as a function of the nanotube diameters. Extracting structural informations at atomic scale with XAS appears as a suitable method. Indeed, Extended X-ray Absorption Fine Structure (EXAFS) studies provide lengths of chemical bonds, coordination numbers with determination of polyiodide species $[13,15,16]$ and could lead to the identification of the inter-chain distances in the case of double and triple chain structures. In association with Raman spectroscopy, x-ray and electron diffraction (ED), these methods provide a complete investigation of the system spanning from local to long range order.

\section{Experimental}

\section{Sample preparation.}

Powders of raw single-wall nanotubes present a distribution in diameter related to the synthesis route. In this study, three samples were selected with mean diameter of $0.9 \mathrm{~nm}, 1.1$ $\mathrm{nm}$ and $1.42 \mathrm{~nm}$ from CoMoCAT (from Aldrich company), HiPCO (from MER company), and electric arc discharge (from NanoCarbLab company) syntheses, respectively. In the following, these samples will be labeled CMT, HiPCO and NCLB, respectively. As described elsewhere [13], nanotube powders were mixed with molten iodine at $120^{\circ} \mathrm{C}$. Doped powders were dried in air for several days and washed with ethanol to remove the excess of weakly bonded iodine. At the end of the procedure, nanotube powders looked similar to the raw material and no iodine, in excess that would induce a texture change or a color change- was observed. The selected nanotube mean diameters should allow one to access to all type of chain structures determined by Guan et al [14], identified as the single, double and triple chain structures.

Raman experiments were performed on a triple monochromator spectrometer (Jobin Yvon T64000), equipped with a charge-coupled detector, in a backscattering geometry, using the $514.5 \mathrm{~nm}$ excitation wavelength. In order to avoid heating of nanotubes and oligomers, the laser power was adjusted at $700 \mu \mathrm{W}$ with a spot diameter of about $3 \mu \mathrm{m}$ using a $50 \mathrm{X}$ objective with a final resolution of about $2 \mathrm{~cm}^{-1}$.

X-ray Diffraction (XRD) experiments were carried out in transmission geometry, using either a Mo source or a $\mathrm{Cu}$ rotating anode generator (Rigaku), delivering a radiation of wavelength $0.711 \AA$ and $1.542 \AA$, respectively. Two-dimensional (2D) scattering patterns were collected either on standard image plates or on a MAR 345 Image plate detector, for exposure durations between $30 \mathrm{~min}$ and several hours. Diffraction profiles were deduced from integration of the intensity along lines or sections. The volume of the powder in the x-ray beam was about 1 $\mathrm{mm}^{3}$.

Electron diffraction experiments were performed on a TOPCON Akashi 002B fitted with a LaB6 gun at an acceleration voltage of $60 \mathrm{keV}$. This voltage reduces the electron damage on 
the nanotubes and increases the diffraction contrast, which is especially important when the signal to noise ratio is weak as it is for carbon nanotubes. Carbon nanotubes were sonicated in ethanol and then dispersed on a lacey carbon TEM grid. Areas where individual ropes of nanotubes were separated from each other by more than $500 \mathrm{~nm}$ were chosen, and selected area diffraction patterns were taken. The selected area aperture diameter projected in the object plane was $500 \mathrm{~nm}$. Diffraction patterns were collected on a detection system optically coupled to a Coolspan Roper (TM) CCD camera. Typical integration times were 40s.

EXAFS experiments were performed on the BM29 beamline at ESRF, Grenoble, France. Samples were measured at the Iodine $\mathrm{K}$ edge $(33169 \mathrm{eV})$ with a double Si 311 monochromator in transmission mode with optimized ionization chambers. Samples were cooled down in a helium cryostat to $20 \mathrm{~K}$ or $50 \mathrm{~K}$, to minimize thermal disorder. For each sample, several spectra have been acquired to improve the signal to noise ratio. EXAFS spectra were extracted after edge calibration [17,18], background removal and edge jump normalization using the ATHENA software.

\section{Results}

Samples were first characterized by Raman spectroscopy. This technique is particularly efficient for SWNT and provides very useful information on both their structural and electronic properties [19-22]. The low frequency region $\left(130-300 \mathrm{~cm}^{-1}\right)$ exhibits the radial breathing modes (RBM) for which the frequency is directly related to the nanotube diameter $\left(\omega\left(\mathrm{cm}^{-1}\right)=\mathrm{A} / \mathrm{d}(\mathrm{nm})+\mathrm{B}\right.$ where $\mathrm{A}$ and $\mathrm{B}$ are two constants) [19-22]. At higher frequencies, the tangential modes ( $\mathrm{G}$ bands) located around $1600 \mathrm{~cm}^{-1}$ give some insights on the semiconducting or metallic character of the nanotubes. Furthermore, all these modes are particularly sensitive to environmental effects and/or charge transfer [23,24], enabling to probe interactions between nanotubes and host species such as polyiodides. Meanwhile, Raman spectroscopy is also used to investigate polyiodide structural properties [25-28], especially in iodine doped carbon nanotubes [13,29-33]. Short polyiodides can be described by the following formula:

$$
\mathrm{mI}_{2}+\mathrm{nI}^{-} \rightarrow \mathrm{I}_{(2 \mathrm{~m}+\mathrm{n})}{ }^{\mathrm{n}-}
$$

All structures give rise to vibrational modes lying in the $100-200 \mathrm{~cm}^{-1}$ frequency range [2528] bands around 105 and $165 \mathrm{~cm}^{-1}$ are generally assigned to the vibrations of the $\mathrm{I}_{3}{ }^{-}$and $\mathrm{I}_{5}{ }^{-}$ chains [13,29-33]. Figure 1 displays the Raman spectra of pristine (line) and doped SWNT (open circles), NCLB (bottom), CMT (middle) and HiPCO (top) respectively. The diameter distributions are $1.4 \pm 0.2 \mathrm{~nm}, 0.9 \pm 0.06 \mathrm{~nm}$ and $1.1 \pm 0.2 \mathrm{~nm}$ for NCLB, CMT and HiPCO SWNT respectively. It is worth mentioning that the diameter distribution of the HiPCO nanotubes is large enough to encapsulate both single and double iodine chains.

After iodine intercalation, the low frequency region clearly exhibits, for HiPCO and CMT samples, two new broad bands around 105 and $160 \mathrm{~cm}^{-1}$, featuring the presence of short polyiodides [13,29-33]. Some modifications of the spectrum are also observed between 150 and $200 \mathrm{~cm}^{-1}$. Previous works on such systems usually reported the vanishing of the RBM and a significant upshift of the $\mathrm{G}$ band (of about $10 \mathrm{~cm}^{-1}$ ) [32,33]. However, $\mathrm{G}$ bands around 1590 $\mathrm{cm}^{-1}$ display only a slight upshift of only $2 \mathrm{~cm}^{-1}$ and the RBM do not look much affected. 
These observations suggest that the charge transfer might be weaker than observed previously [32].

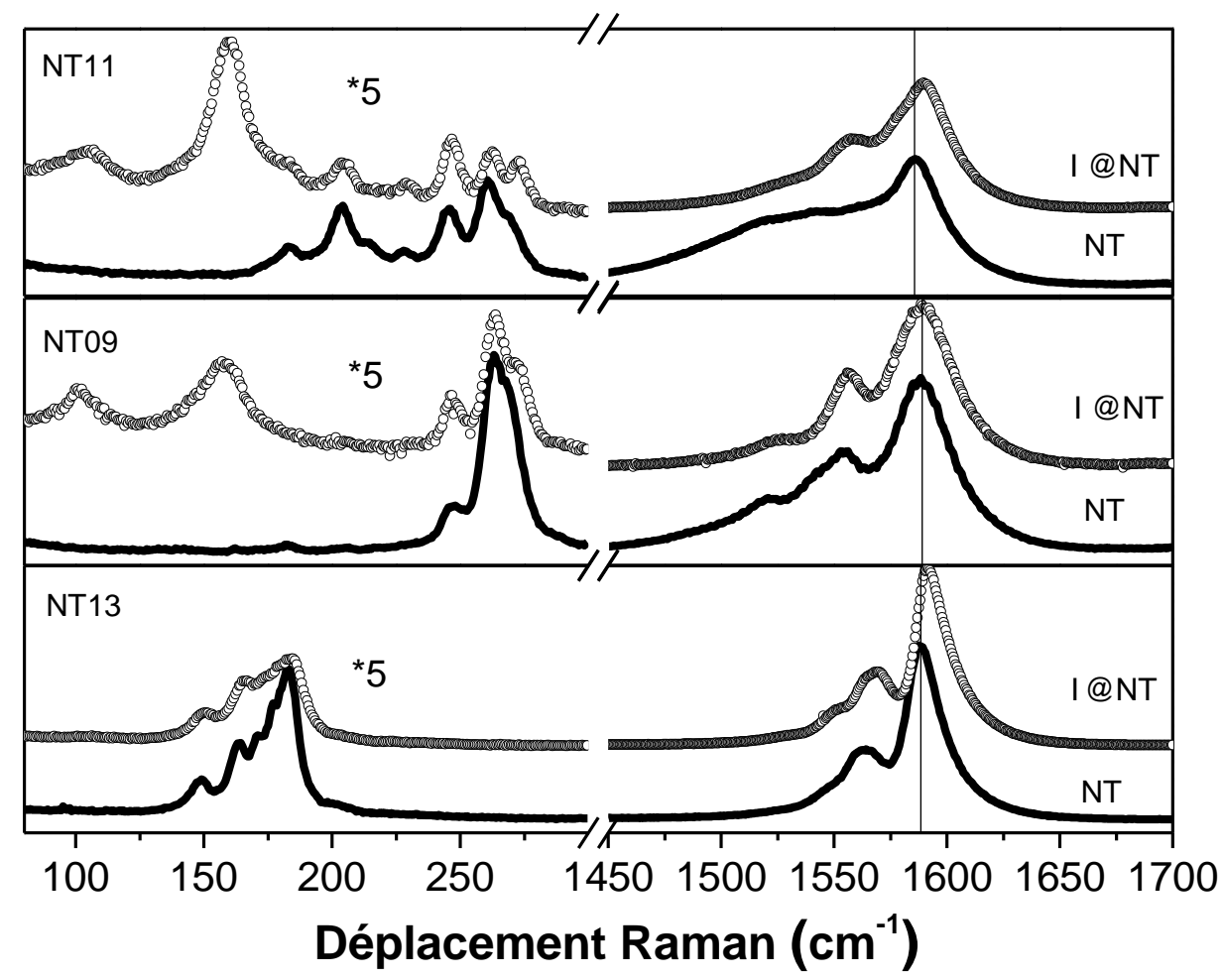

Figure 1: Raman spectra recorded at 514nm for empty (line) and doped (open circle) HiPCO, CMT and NCLB nanotubes, from top to bottom respectively. RBM and iodine modes are located at low energy and nanotube tangential modes are closed to $1600 \mathrm{~cm}^{-1}$.

X-ray diffraction is known to provide a statistically relevant structural characterization of carbon nanotube powders $[34,35]$. SWNT are usually aggregated into hexagonal arrays (the so-called "bundles"), these two-dimensional arrangements giving rise to Bragg scattering. Analysis of the modulations of these Bragg peaks with the form factor of cylindrical-shaped SWNT allows structural parameters to be determined. Among them, let us cite the mean diameter, the width of the diameter distribution, and the number of tubes per bundle. When objects are inserted inside SWNT (e.g. $\mathrm{C}_{60}$ [36], $\mathrm{C}_{70}$ [37], iodine molecules [12]...), the relative intensities and the position of the Bragg peak may significantly vary. Besides, the structure of the guest molecules can generate additional diffusion [36,37,12], as will be discussed below. In the case of electron diffraction, more local examination is performed, for only one nanotube bundle. The obtained scattering pattern presents an intense equatorial line, whose modulations are related to the hexagonal arrangement of nanotubes. Other diffuse lines 
parallel to the equatorial lines, remanent from the zig-zag arrangement of the graphene lattice, are also visible, the maxima of which form circle arcs. Moreover, one-dimensional periodic chains of molecules inside nanotubes lead to characteristic diffuse lines, corresponding to the intersection of the corresponding diffuse planes with the Ewald sphere, as was shown for instance for fullerenes [38]. Both statistical and local approaches were combined here, for the CMT and HiPCO samples.

Figure 2 presents the XRD pattern obtained for pristine and doped HiPCO, and doped CMT, while calculated diffraction patterns are shown in Figure 3.

Typical scattering pattern of pristine nanotubes (Figure 2, curve a) shows weak peaks originating from their hexagonal packing in bundles. The HiPCO diffraction profile is satisfactorily reproduced following the formalism detailed in ref. [35], with $\sim 10$ tubes per bundle, mean tube diameter is about $1 \mathrm{~nm}$ with a diameter distribution of Full Width at Half Maximum (FWHM) $0.2 \mathrm{~nm}$ (see Figure 3 for a filling rate $\mathrm{p}=0$ ).

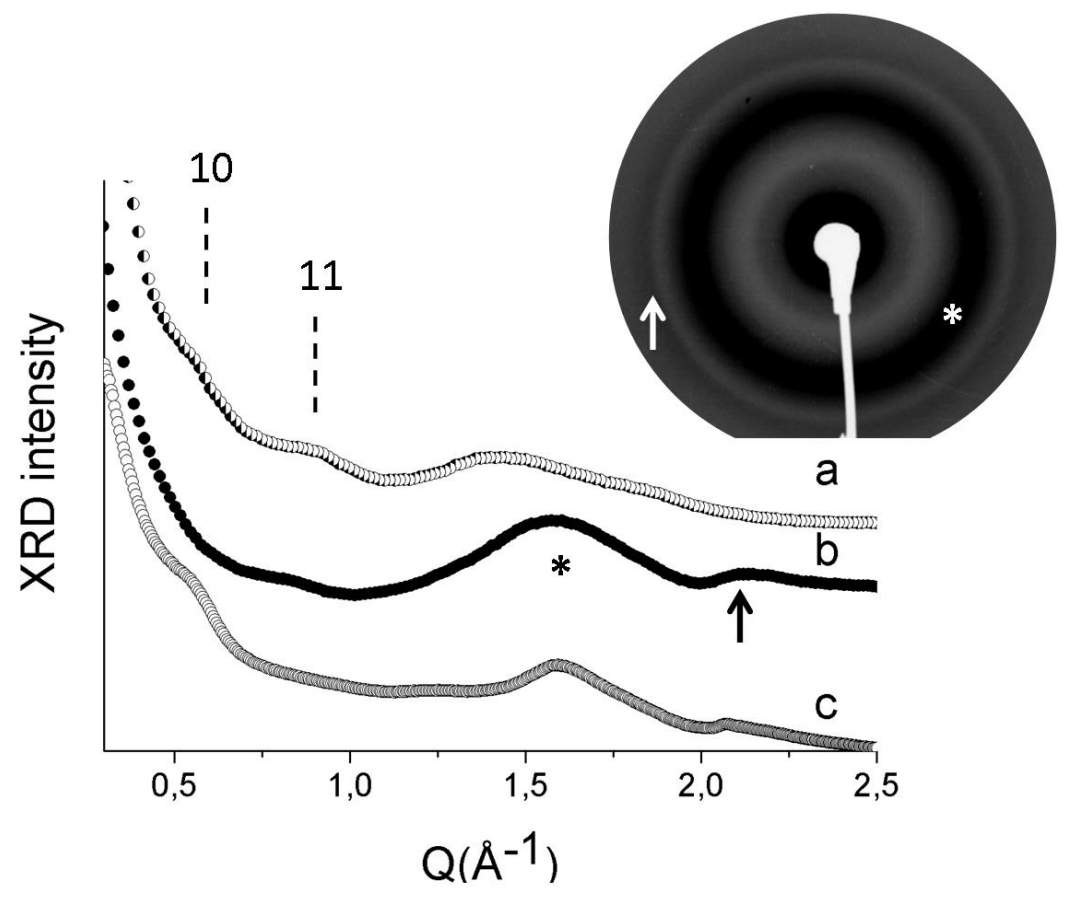

Figure 2: XRD intensity from powders of (a) HiPCO, (b) iodine doped HiPCO and (c) iodine doped CMT nanotubes. HiPCO and CMT diffraction patterns have been obtained with a Mo and $a \mathrm{Cu}$ anode, respectively. Inset: $x$-ray scattering pattern of iodine doped HiPCO as recorded on image plate. Dotted lines indicate hk peaks from the hexagonal bundle lattice, the star and the arrow show additional scattering features observed in iodine doped nanotubes. 


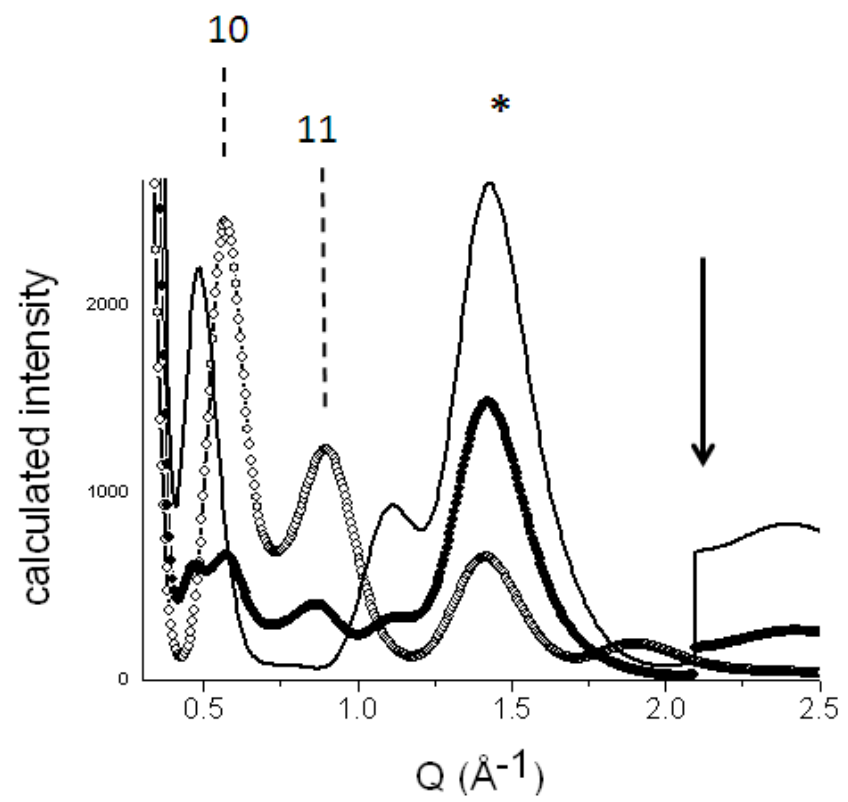

Figure 3: Calculated XRD intensity for iodine doped HiPCO nanotubes, depending of the filling ratio $p$ of the nanotubes. Nanotubes are organized in bundles of 13 tubes, their mean diameter is $1 \mathrm{~nm}$, assuming a Gaussian distribution of diameter with $F W H M=0.2 \mathrm{~nm}$. Open circles correspond to $p=0$ (empty tubes), filled ones to $p=0.5$ and the full line to $p=1$. For the 10 and 11 peaks, the star and the arrow: see Figure 2 caption.

In iodine doped HiPCO scattering patterns, new features appear compared to those of pristine nanotubes (see the star and the arrow in Figure 2). The large peak centered on $1.6 \AA^{-1}$ (indicated with a star) has already been observed in previous XRD investigations on iodinedoped SWNT [12]. Bendiab et al. attributed it to first-neighbor distances between iodine and carbon atoms (estimated to $\sim 4 \AA$ ) revealing the intra-tube encapsulation of iodine atoms. Besides a second feature located around $2.1 \AA^{-1}$ is observed in the HiPCO diffraction profile and highlighted with an arrow on Figure 3. It has never been observed in previous XRD investigations. A closer look to the image plate (inset in Figure 2) allows us to precise the original asymmetric shape of this feature, appearing sharp at its low angle side and smooth at its high angle side. This "sawtooth" shape is typical of 1D periodicity and has already been observed in the case of $\mathrm{C}_{60}$ or $\mathrm{C}_{70}$ molecular arrays in SWNT [36,37].

In the case of doped CMT nanotubes, a similar sawtooth shape peak is observed in Figure 2 at $2.07 \AA^{-1}$, pointing out the relevance of $1 \mathrm{D}$ arrangement of iodine in such narrow SWNT. The wave-vector value $\mathrm{Q} \sim 2.1 \AA^{-1}$ corresponds to a mean distance between iodine ions of $\mathrm{d} \sim 3 \AA$ $(\mathrm{Q}=2 \pi / \mathrm{d})$, in good agreement with the length of the ionic bond in asymmetric triiodide $\mathrm{I}_{3}{ }^{-}$and in the pentaiodide $\mathrm{I}_{5}^{-}$[39].

Finally, one could also note that relative intensities of the hk peaks are different for pristine and doped HiPCO samples: the relative scattering intensity 10 peak appears smaller in iodine doped sample which is related to iodine filling. To validate this statement, theoretical XRD patterns were calculated following the formalism developed for fullerenes previously [36,37]. Results are shown in Figure 3, using the form factor of iodine instead of fullerenes and taking an inter-iodine distance of $3 \AA$. Upon doping, the intensity of the 10 peak drops up to a filling rate of $\mathrm{p} \sim 0.5$ and then rises again. The broad peak around $1.5 \AA^{-1}$ as well as the saw-tooth one at $\sim 2.1 \AA^{-1}$ increase with the filling rate p. With this simple model used here, qualitative agreement with experimental data is thus found for $\mathrm{p} \sim 0.5$ : the large and intense feature around $1.6 \AA^{-1}$ is slightly down-shifted, but the asymmetric peak at $2.07 \AA^{-1}$, which is the first 
XRD evidence for the one-dimensional long range ordered structure of iodine inside SWNT, is correctly simulated.

Electron diffraction patterns, such as the one shown in Figure 4, corroborated the XRD results. The equatorial line and the circle arc mentioned above are seen. Strong modulations along the equatorial axis linked to the hexagonal arrangement of the tubes in the bundle are also observed. In addition, to these regular SWNT ropes informations, the diffuse line, corresponding to a periodicity of $3 \AA$, is clearly observed.

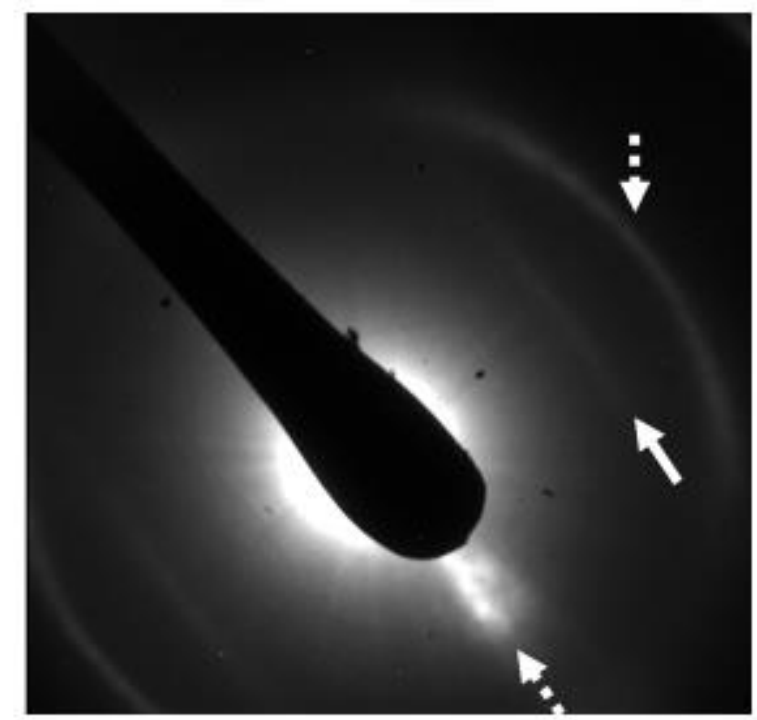

Figure 4: Electron diffraction pattern of a iodine doped nanotube bundle. Dotted arrows point towards nanotube typical features (the equatorial plane as well as the ring aroung 2.9A-1 corresponding to the 10 peak from graphene sheets) while the solid arrow points toward a diffuse line at about $2 \AA^{-1}$, corresponding to the inter-iode distance of $3 \AA$.

Figure 5 displays the EXAFS spectra measured on CMT, HiPCO, and NCLB samples. The $\mathrm{k}^{3}$ weight is applied to make the high-k oscillations visible. Spectra are well defined up to $\mathrm{k}=18$ $\AA^{-1}$ and exhibit only slight differences. This observation is surprising as significant changes should be expected in the spectra due to the different chain structures (single to triple) reported in the literature [14]. The corresponding Fourier transforms are performed with a Hanning window $(\mathrm{dk}=0.2)$ in the $\mathrm{k}$ range from 2.3 to $16.9 \AA^{-1}$. These Fourier transforms, representing the pseudo radial density function around the absorbing atoms, are composed of two peaks located at $2.8 \AA$ and $5.8 \AA$ (without phase shift correction), related to the first and second atomic shells.

In Figure 5 the upper spectrum represents a FEFF 6 simulation on the structural model used in the diffraction analysis. We consider a chain of iodine atoms with a lattice parameter of $3 \AA$. The scattering paths which generate the two peaks visible in the right panel of Figure 5 can be detailed as follow. The first peak is related to the distance between the absorber and the first neighbor at $3 \AA$ (first shell). This signal is generated by a single scattering path. The second peak corresponds to the distance to the second neighbors at $6 \AA$ (second shell). The amplitude of this peak is given mainly by two multiple scattering paths (so-called 3 legs and 4 legs) five times more intense than the second neighbor single scattering path. Amplitudes of the 3 legs and 4 legs are enhanced due to the focusing effect due to the alignment of the atoms. The 
presence of the second peak is the key point that characterizes the one dimensionality of the system at the local atomic scale.

One can observe that the experimental Fourier Transforms are comparable to the simulated one. The second peak is always present in the experimental, data evidencing the local one dimensionality of the iodine system in the carbon nanotubes. However the magnitude and location of the peaks differ from the calculated ones. This behavior implies that the chain model satisfying a first approach to interpret diffraction experiments, has to be improved to satisfy the local order.
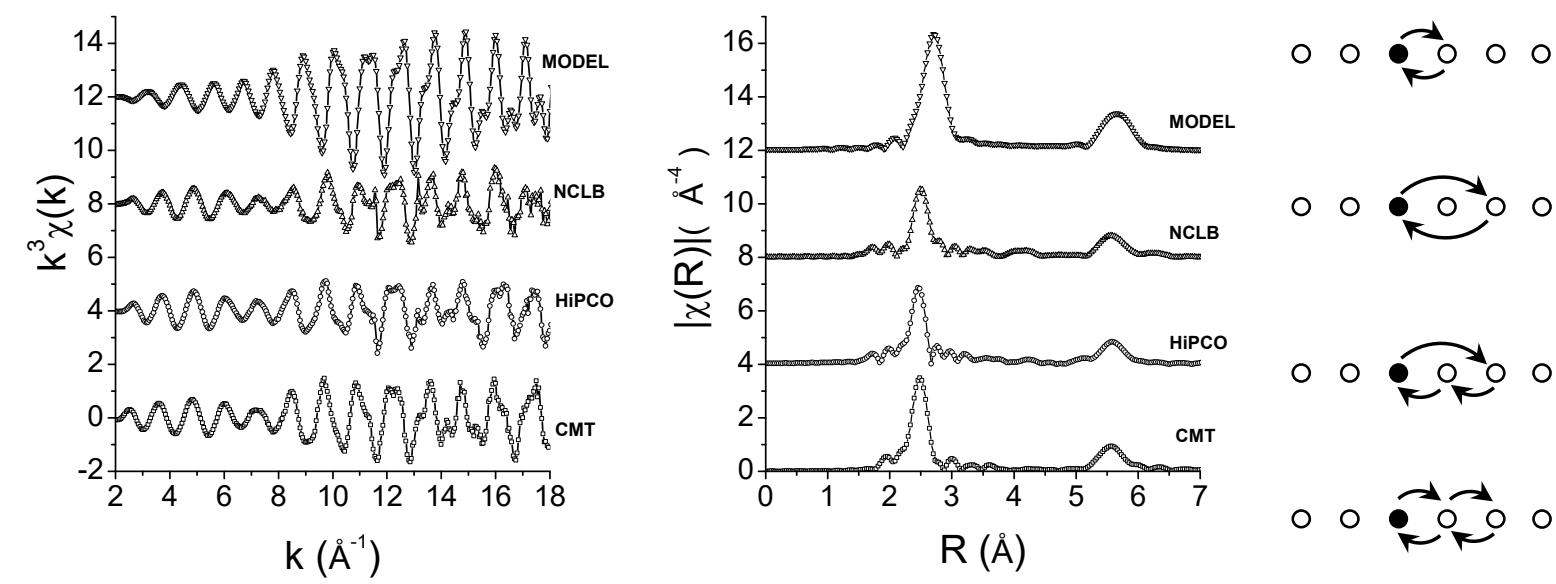

Figure 5: Left: experimental EXAFS spectrum measured at 50K for NCLB and HiPCO samples and $20 \mathrm{~K}$ for CMT sample. The upper spectrum is a simulation for a pefect chain of aligned iodine atoms with a constant step of $3 \AA$. Center: Corresponding EXAFS Fourier Transforms. Right: Schematic description of the scattering paths involved in the chain model for the first shell and second shell signals. From top to bottom, first shell single scattering, second shell single scattering, 3 legs and 4 legs multiple scattering. The black dot represents the absorber and white dots are the scatterers.

Let us consider the detailed analysis of the first shell peak. It was first performed using the ARTEMIS software. This software requires a good knowledge of the structure of the studied material in order to initialize correctly the fit parameters and to obtain reliable physical informations as inter-neighbor distances $\mathrm{R}$, coordination numbers $\mathrm{N}$, mean square relative displacement $\sigma^{2}$ (msrd). We thus built different models of iodine chains containing from one to four distances and one more to consider the scattering from the carbon nanotube walls. Unfortunately, analysis did not converge to a unique and physically reasonable model. Indeed fit parameters were too strongly correlated to each others which led to the divergence of the errors bars. In order to avoid this issue, we thus used the Landweber iteration method for EXAFS described by Rossberg and Funke in ref [40]. With this method the radial particle distribution function (RPDF) is calculated directly by inverting the EXAFS integral equation [40]. Opposite to conventional shell fitting approaches, no predefinition of the RPDF peak shape must be done to initialize the fit. This method is suitable for a calculation of the RPDF of one type of backscattering atom but can be efficiently extended to two types of 
backscattering atoms. The detailed mathematical description of this extension will be the content of a following paper. The RPDF is calculated in an interval of 2.5 to $3.6 \AA$ with a step of $0.01 \AA$ for both iodine and carbon shells. The optimized number of iterations $v$ is defined according to the study of the L-curve as described in ref [40]. After iteration the extracted RPDF is analyzed in order to find a best description with single shells. The best description of the RPDF's is obtained by using Gaussian functions (Figure 6). Each Gaussian corresponds to a single shell of atoms for which the structural parameter R, N and $\sigma^{2}$ are accessible. For each shell, the distance $\mathrm{R}$ is given by the center of the Gaussian, the mean square displacement $\sigma^{2}$ is given by the FWHM and the coordination number $\mathrm{N}$ is given by the total number of atoms in the shell [40]. The results are summarized in table 1.
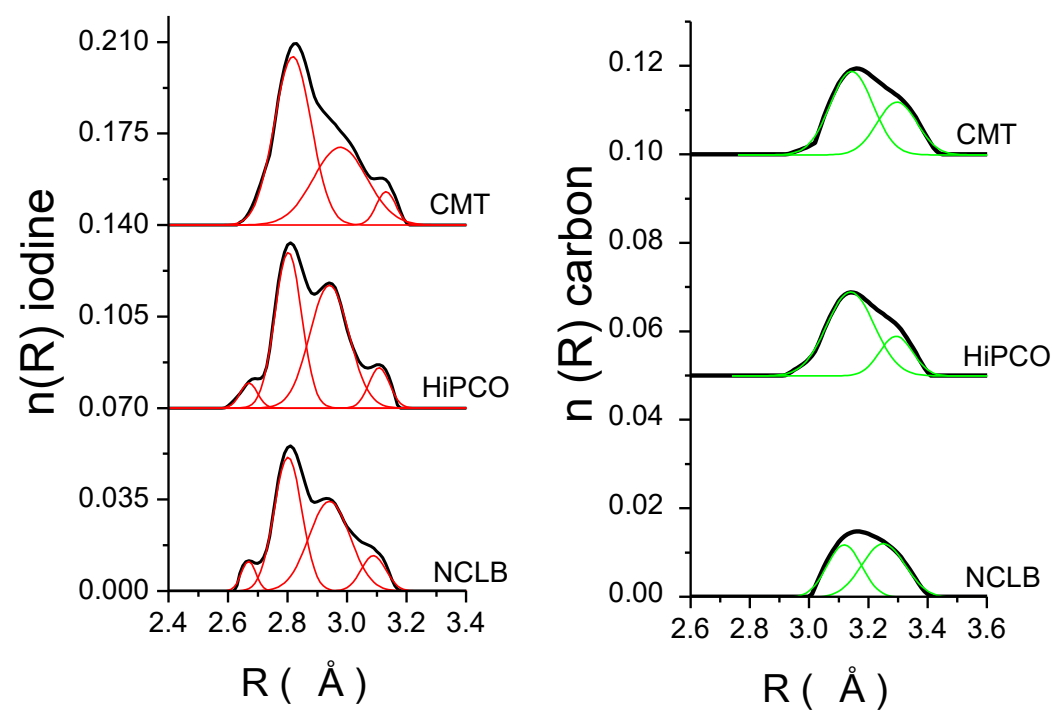

Figure 6: Analysis of the iodine and carbon RPDF obtained with the Landweber iteration method. Structural parameters for each contribution are summarized in table 1.

For all samples, iodine RPDF contains three distinct components of about $2.8 \AA, 2.94 \AA$ and $3.1 \AA$. For HiPCO and NCLB a fourth distance at $2.66 \AA$ is also present but it corresponds to a small coordination number $(\mathrm{N}=0.07)$. The carbon RPDF are centered on $3.2 \AA$ which is in agreement with the distance between the iodine atom and the carbon nanotube wall already observed [10,11].

The inter atomic distances of $2.8 \AA$ and $3.1 \AA$ are known to correspond respectively to the covalent bond of diiodine $I_{2}$ and to the ionic bond in polyiodides (see eq. (1)). The interatomic distance of $2.94 \AA$ can be assigned to the typical covalent bond of the symmetric triiodide $\mathrm{I}_{3}{ }^{-}$[39]. The analysis of the first shell peak therefore reveals a mixture of polyiodides inside the nanotubes. Chains are formed by consecutive short linear polyiodides $\mathrm{I}_{3}{ }^{-}$ (symmetric and asymmetric), $\mathrm{I}_{5}{ }^{-}$and probably longer linear polyiodides $\mathrm{I}_{\mathrm{n}}{ }^{-}$in agreement with our XRD results which show the existence of an average distance of about $3 \AA$ between iodine atoms and/or ions. Note finally that, excepted symmetric $\mathrm{I}_{3}^{-}$, all linear polyiodides $\mathrm{I}_{n}^{-}$ contain molecular and ionic bonds. The coordination numbers associated to these bonds are thus averaged over all these polyiodides species, rendering impossible the quantitative determination of the $\mathrm{I}_{\mathrm{n}}{ }^{-}$distribution. 


\begin{tabular}{|c|c|c|c|c|c|c|c|c|c|c|c|c|c|c|c|}
\hline & \multicolumn{3}{|c|}{$\begin{array}{l}\text { lodine } \\
\text { 1st shell }\end{array}$} & \multicolumn{3}{|c|}{$\begin{array}{l}\text { lodine } \\
\text { 2nd shell }\end{array}$} & \multicolumn{3}{|c|}{$\begin{array}{l}\text { lodine } \\
\text { 3rd shell }\end{array}$} & \multicolumn{3}{|c|}{$\begin{array}{l}\text { Carbon } \\
\text { 1st shell }\end{array}$} & \multicolumn{3}{|c|}{$\begin{array}{c}\text { Carbon } \\
\text { 2nd shell }\end{array}$} \\
\hline & $\mathrm{R}$ & $\mathrm{N}$ & $\begin{array}{c}\sigma^{2} \\
\left({ }^{*} 10^{-3}\right)\end{array}$ & $\mathrm{R}$ & $N$ & $\begin{array}{c}\sigma^{2} \\
\left(* 10^{-3}\right)\end{array}$ & $\mathrm{R}$ & $\mathrm{N}$ & $\begin{array}{c}\sigma^{2} \\
\left({ }^{*} 10^{-3}\right)\end{array}$ & $\mathrm{R}$ & $\mathrm{N}$ & $\begin{array}{c}\sigma^{2} \\
\left(* 10^{-3}\right)\end{array}$ & $\mathrm{R}$ & $N$ & $\begin{array}{c}\sigma^{2} \\
\left(* 10^{-3}\right)\end{array}$ \\
\hline CMT & 2.817 & 1.00 & 3.8 & 2.977 & 0.66 & 6.0 & 3.131 & 0.10 & 1.0 & 3.143 & 0.327 & 5.05 & 3.296 & 0.20 & 4.4 \\
\hline HPCO & 2.802 & 0.67 & 2.0 & 2.940 & 0.77 & 4.3 & 3.108 & 0.134 & 1.20 & 3.140 & 0.372 & 6.30 & 3.293 & 0.116 & 3.0 \\
\hline NCLB & 2.801 & 0.59 & 2.2 & 2.941 & 0.59 & 4.8 & 3.090 & 0.140 & 1.70 & 3.120 & 0.158 & 3.24 & 3.25 & 0.207 & 5.10 \\
\hline
\end{tabular}

Table1: Structural parameters, distances $R(\AA$,$) , mean square relative displacement \sigma^{2}\left(\AA^{2}\right)$ and coordination number $N$, for the iodine and carbon shells from the Landweber iteration method.

The quantitative analysis of the experimental second peak at $5.8 \AA$ is less straightforward to achieve. To the best of our knowledge the fit of such peak has never been performed. Indeed the observation of this peak is not systematic [13,15] or is reported with much lower magnitude [16 and ref therein]. 

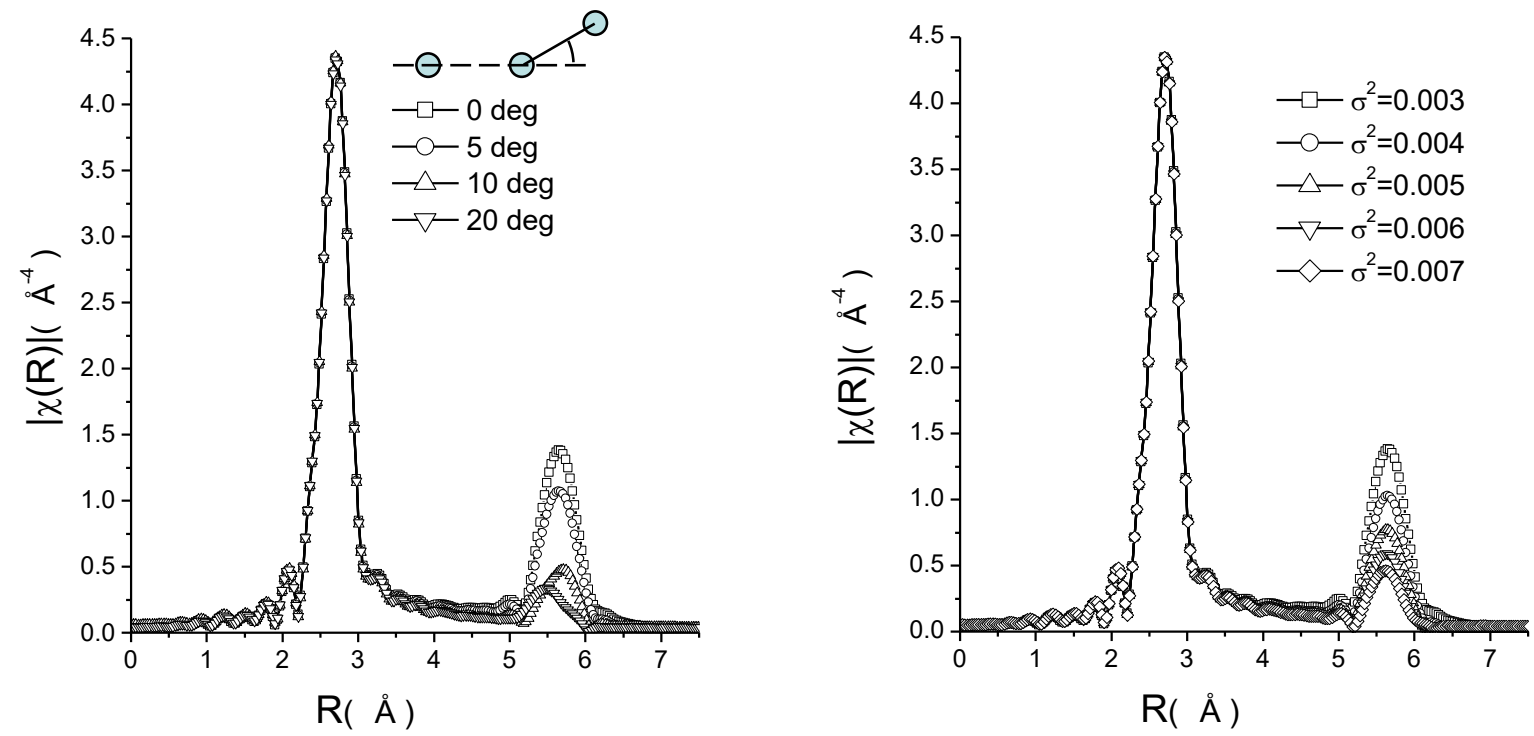

Figure 7: Simulations of the second shell peak amplitude as a function of the alignment of the atoms in the chain (left) and of their total mean square relative displacement (right).

As previously discussed, this signal comes predominantly from the 3 legs and 4 legs multiple scattering paths generated by at least three consecutive and aligned atoms (fig. 5). The amplitude of the signal strongly depends on two main factors: the alignment of the atoms and the mean square displacement of the atoms involved in the multiple scattering paths. In order to evaluate qualitatively the effect of these parameters, we performed some simulations with Artemis software (see fig 7).

Firstly, by referring to Figure $7 \mathrm{a}$, one can see that the amplitude of the second shell peak is sensitive to the alignment of the atoms. Amplitude of about $1 \AA^{-4}$ being observed experimentally (Figure 5), misalignment should not exceed 5 degrees. For larger angles, focusing effect that enhances the amplitude disappears and the signal is dramatically reduced. As EXAFS provides the averaged local structure of an atomic species in a sample, one can say that, the overall of iodine atoms in the nanotubes have a quasi linear local environment. The direct implication is that the overall of molecules in the nanotubes are rather linear.

Secondly, considering the amplitude of about $1 \AA^{-4}$ observed experimentally in all samples, total msrd of the multiple scattering paths is found to be about $0.005 \AA^{2}$. The reader may note that previous EXAFS studies on polyiodides $[13,15,16]$ mentioned that ionic bond has large msrd which kills the amplitude of the second shell peak. A direct consequence is that no second shell peak can be observed from linear $\mathrm{I}_{n}^{-}$polyiodides containing ionic bonds $[13,15,16]$. However, it is worth noting that within the assumption that only symmetric $\mathrm{I}_{3}{ }^{-}$ species contribute to the second peak [16], we failed to fit it with the associated multiple scattering paths. Therefore, more paths have to be added. Such multiple scattering paths could be generated in long $\mathrm{I}_{\mathrm{n}}{ }^{-}$polyiodides i.e. from atoms of ionic bounds or of consecutive molecular iodine. This result corroborates XRD experiments: a long range one dimensional ordering of the polyiodides is stabilized by the confinement in the nanotubes.

The qualitative analysis of the second shell peak gives important informations on the one dimensional ordering of the polyiodides inside carbon nanotubes. It shows that most of the polyiodides are linear and that they appear aligned and organized over long distances. 
Let us now discuss about the discrimination between the different chain structures proposed in the literature [14]. The mean diameters of the nanotubes have been selected in order to reach the different chain structures according to Guan et. al. [14]. The CMT, HiPCO and NCLB samples should contain single, single and double, and triple chain structures respectively. The second peak observed in our EXAFS experiments is a typical signature of an intra-chain distance and not of an inter chain distance. The inter-chain distances for the double chain was estimated to $4.9 \AA$ after Guan et. al.. The Fourier transform of the samples HiPCO and NCLB should consequently show an additional peak relative to this distance situated in between the first shell peak and the second shell peak. However such signature is not present in the Fourier transform spectra. It can be explain as follow. First the inter chain distance could have a large dispersion, inducing a smearing of the peak in the Fourier transform. Indeed, it has been observed that the interactions of two and three chains with the nanotube wall create deformation of the carbon wall and of the chains themselves [14]. Moreover, double and the triple helix structures sometimes observed do not have a constant pitch. Secondly, the inter chains distance could be slightly larger than the value estimated to $4.9 \AA$. Then the amplitude of the EXAFS signal, generated by a single scattering path should be weak and merge into the second shell peak. We want to point out the fact that although the inter-chain signal cannot be detected in our EXAFS, this does not imply that multiple chain structures are not present in the nanotubes.

EXAFS analysis appears as a useful tool to quantitatively solve the first shell coordination of the long chains of polyiodides confined inside carbon nanotubes. This analysis evidences the mixing of different structures of polyiodides. The second shell peak provides more qualitative information but it nevertheless points toward iodine molecule alignment along the chain.

\section{Conclusion}

This work gives experimental proofs of the 1D-confinement of short polyiodide species inside the hollow core of single-walled carbon nanotubes. Long range one dimensional ordering of the iodine species is shown by x-ray and electron diffraction experiments independently of the tube diameter. Quantitative analysis of the local arrangement of iodine species inside the nanotubes by x-ray absorption spectroscopy reveals the presence of confined linear structures of diiodine $\left(\mathrm{I}_{2}\right)$, triodide $\left(\mathrm{I}_{3}^{-}\right)$and pentaiodide $\left(\mathrm{I}_{5}^{-}\right)$. 
Bibliography :

[1] S. Iijima, Nature 354, 56 (1991)

[2] Z. Wu, Z. Chen, X. Du, J. M.Logan, J. Sippel, M. Nikolou, K. Kamaras, J. R. Reynolds, D. B. Tanner, A. F. Hebard and A. G. Rinzler, Science, 305,1273, 2004

[3] P. Jarillo-Herrero, J. Van Dam, L. Kouwenhoven, Nature, 439, 953, (2006)

[4] D. M. Guldi, Nature, 447, 50, (2007)

[5] R.S. Lee, H. J. Kim, J. E. Fischer, A. Thess, and R. E. Smalley, Conductivity enhancement in single-wall carbon nanotube bundles doped with $\mathrm{K}$ and $\mathrm{Br}$, Nature (London) 388, 257 (1997)

[6] A. S. Claye, N. M. Nemes, A. Janossy, and J. E. Fischer, Structure and electronic properties of potassium doped single-wall carbon nanotubes, PRB 62, R4845 (2000).

[7] X. Liu, T. Pichler, M. Knupfer, and J. Fink, Electronic and optical properties of alkali metal intercalated single-wall carbon nanotubes, PRB 67, 125403 (2003).

[8] L. Duclaux, J. P. Salvetat, P. Lauginie, T. Cacciaguera, A. M. Faugre, C. Goze-Bac, and P. Bernier, Synthesis and characterization of SWNT-heavy alkali metal intercalation compounds, effect of host SWNTs materials, J. Phys. Chem Solids 64, 571, (2003).

[9] L.Grigorian, K. A. Wiliams, S. Fang, G. U. Sumanasekera, A. L. Loper, E. C. Dickey, S. J. Pennycook, and P. C. Eklund, Reversible intercalation of chargerd Iodine chains into carbon nanotube ropes, Phys. Rev. Lett, 80, (1998), 5560.

[10] P. C. Eklund, L. Grigorian, K. A. Williams, G. U. Sumanasekera, S. Fang, Metallic nanoscale fibers from stable iodine-doped carbon nanotubes, United States Patent, 6139919, (2000).

[11] X. Fan, E. C. Dickey, P. C. Eklund, K. A. Williams, L. Grogorian, R. Buczko, T. T. Pantelides, and S. J. Pennycook, Atomic arrangement of iodine inside single-wall carbon nanotubes, Phys. Rev. Lett 84, 4621, (1998).

[12] N. Bendiab, R. Almairac, S. Rols. R. Aznar, and J. L. Sauvajol, I. Mirebeau, Structural determination of iodine localization in single-wall carbon nanotubes bundles by diffraction methods, Phys. Rev. B 69, 195415, (2004).

[13] T. Michel, L. Alvarez, J. L. Sauvajol, R. Almairac, R. Aznar, and J. L. Bantignies, O. Mathon, EXAFS investigations of iodine-doped carbon nanotubes, Phys. Rev. B 73, 195419, (2006).

[14] L. Guan, K. Suenaga, Z. Shi, Z. Gu, and S. Iijima, Polymorphic structures of iodine and their phase transition in confined nanospace, Nanoletters (2007), 7, 1532. 
[15] K.E. Aasmundveit, E. J. Samuelsen, C. Steinsland, C. Meneghini and A. Filipponi, EXAFS study of iodine doped poly(octylthiophene), Synthetic Metals, 101, 363, 1999.

[16] H. Sakane, T. Mitsu, H, Tanida, and I. Watanabe, XAFS analysis of triiodide ion in solution, J. Sync, Rad, 8, 674, 2001

[17] J. Padeznik Gomilsek, I. Arcon, S. de Panfilis, and A. Kodre, X-ray absorption coefficient of iodine in the K edge region, J. Phys. B : At. Mol. Opt. Phys. 41, 025003, (2008)

[18] J. Padeznik Gomilsek, I. Arcon, S. de Panfilis, and A. Kodre, X-ray absorption in atomic iodine in the K edge region, Phys. Rev. A, 79, 032514, (2009)

[19] M. S. Dresselhaus, G. Dresselhaus, R. Saito, A. Jorio, Physics reports, 409, 47, 2005

[20] C.Thomsen, S. Reich, J. Maultzch, Phil. Trans. R. Society, Lond. A, 362, 2337, (2004),

[21] J.L. Sauvajol, E. Anglaret, S. Rols, L. Alvarez, Carbon, 40, 1697-1714, (2002)

[22] P.T. Aurojo, P. B. C. Pesce, M. S. Dresselhaus, K. Sato, R. Saito, A. Jorio, Physica E, $42,1251,(2010)$

[23] J. C. Tang, nature nanotechnology, 2, 725, 2007

[24] M. Kalbac, H. Farhat, L. Kavan, J. Kong, M. S. Dresselhaus, Nano Letters 8 (10):353235372008

[25] P.Deplano, F. Devillanova, J. Ferraro, M.L. Mercuri, V. Lippolis and E. Trogu, Applied Spectroscopy, 48, 1236, (1994),

[26] R. Teitelbaum, S. Ruby and T. Marks, JACS, 7568, (1979)

[27] P. Deplano, J. Ferraro, M.L. Mercuri and E. Trogu, Coordination Chemestry Reviews, $188,71,(1999)$

[28] S. Hsu, A. Signorelli, G. Pez, R. Baughman, J. Chem. Phys, 69, 106, (1978)

[29] L. Grigorian, K.A. Williams, S. Fang, G.U. Sumanasekera et al. Phys. Rev. Lett., 80, 5560, (1998)

[30] W. Zhou, S. Xie, L. Sun, D. Tang et al, et al, App. Phys. Lett., 80, 2553, (2002)

[31] U.D. Venkateswaran, E.A. Brandsen, M. Katakowski, A. Harutyunyan et al., Phys. Rev.B, 65, 054102, (2002)

[32] T. Michel , L. Alvarez, JL Sauvajol, R. Almairac, et al, J. of Phys. and Chem. of Solids,

[33] L. Alvarez et al, Physical Review B, Vol.82, 205403, 2010 
[34] A. Thess, R. Lee, P. Nikolaev, H. Dai, P. Petit, J. Robert, C. Xu, Y. Hee Lee, S. Gon Kim, A.G. Rinzler, D.T. Colbert, G.E. Scuseria, D. Tomanek, J.E. Fischer, and R.E. Smalley, Crystalline ropes of metallic carbon nanotubes, Science 273, 56 (1995)

[35] S. Rols, R. Almairac, L. Henrard, E. Anglaret and J.L. Sauvajol, Eur. Phys. J. B 10, 263 (1999)

[36] J. Cambedouzou, V. Pichot, S. Rols, P. Launois, P. Petit, R. Klement, H. Kataura, and R. Almairac, On the diffraction pattern of C60 peapods, Eur. Phys. J. B 42, 31 (2004)

[37] M. Chorro, A. Delhey, L. Noé, M. Monthioux and P. Launois, Orientation of C70 molecules in peapods as a function of the nanotube diameter, Phys. Rev. B 75, 035416 (2007)

[38] K.Hirahara, S. Bandow, K. Suenaga, H.Kato, T. Okazaki, H. Shinohara, and S. Ijima, Electron diffraction study of one-dimensional crystals of fullerenes , Phys. Rev. B 64, 115420 (2001).

[39] P. H. Svensson and L. Kloo, Synthesis, Structure, and bonding in polyiodide and metal iodide-iodine Systems, Chem. Rev. 103, 1649, 2002

[40] A. Rossberg and H. Funke, Determining the radial pair distribution function from X-ray absorption spectra by use of the Landweber iteration method, Journal of Synchrotron Radiation, 17, 280, 2010 